\title{
Diffusion Approximations of the Geometric Markov Renewal Processes and Option Price Formulas
}

\author{
Anatoliy Swishchuk ${ }^{\mathbf{1}}$ and M. Shafiqul Islam ${ }^{2}$ \\ ${ }^{1}$ Department of Mathematics and Statistics, University of Calgary, 2500 University Drive, NW, Calgary, \\ Alberta, Canada T2N 1N4 \\ ${ }^{2}$ Department of Mathematics and Statistics, University of Prince Edward Island, 550 University Avenue, \\ Charlottetown, PE, Canada C1A 4P3
}

Correspondence should be addressed to M. Shafiqul Islam, sislam@upei.ca

Received 3 August 2010; Accepted 8 November 2010

Academic Editor: Aihua Xia

Copyright (c) 2010 A. Swishchuk and M. S. Islam. This is an open access article distributed under the Creative Commons Attribution License, which permits unrestricted use, distribution, and reproduction in any medium, provided the original work is properly cited.

We consider the geometric Markov renewal processes as a model for a security market and study this processes in a diffusion approximation scheme. Weak convergence analysis and rates of convergence of ergodic geometric Markov renewal processes in diffusion scheme are presented. We present European call option pricing formulas in the case of ergodic, double-averaged, and merged diffusion geometric Markov renewal processes.

\section{Introduction}

Let $N(t)$ be a standard Poisson process and $\left(Y_{k}\right)_{k \in Z_{+}}$be i.i.d. random variables which are independent of $N(t)$ and $S_{0}^{*}>0$. The geometric compound Poisson processes

$$
S_{t}^{*}=S_{0}^{*} \prod_{k=1}^{N(t)}\left(1+Y_{k}\right), \quad t>0,
$$

is a trading model in many financial applications with pure jumps [1, page 214]. Motivated by the geometric compound Poisson processes (1.1), Swishchuk and Islam [2] studied the Geometric Markov renewal processes (2.5) (see Section 2) for a security market in a series scheme. The geometric Markov renewal processes (2.5) are also known as a switched-switching process. Averaging and diffusion approximation methods are important 
approximation methods for a switched-switching system. Averaging schemes of the geometric Markov renewal processes (2.5) were studied in [2].

The singular perturbation technique of a reducible invertible-operator is one of the techniques for the construction of averaging and diffusion schemes for a switchedswitching process. Strong ergodicity assumption for the switching process means that the singular perturbation problem has a solution with some additional nonrestrictive conditions. Averaging and diffusion approximation schemes for switched-switching processes in the form of random evolutions were studied in [3, page 157] and [1, page 41]. In this paper, we introduce diffusion approximation of the geometric Markov renewal processes. We study a discrete Markov-modulated $(B, S)$-security market described by a geometric Markov renewal process (GMRP). Weak convergence analysis and rates of convergence of ergodic geometric Markov renewal processes in diffusion scheme are presented. We present European call option pricing formulas in the case of ergodic, double-averaged, and merged diffusion geometric Markov renewal processes.

The paper is organized as follows. In Section 2 we review the definition of the geometric Markov renewal processes (GMRP) from [2]. Moreover we present notation and summarize results such as random evolution of GMRP, Markov renewal equation for GMRP, infinitesimal operator of GMRP, and martingale property of GMRP. In Section 3 we present diffusion approximation of GMRP in ergodic, merged, and double-averaging schemes. In Section 4 we present proofs of the above-mentioned results. Section 4 contains solution of martingale problem, weak convergence, rates of convergence for GMRP, and characterization of the limit measure. In Section 5 we present merged diffusion GMRP in the case of two ergodic classes. European call option pricing formula for ergodic, merged, and diffusion GMRP are presented in Section 6.

\section{The Geometric Markov Renewal Processes (GMRP)}

In this section we present the Geometric Markov renewal processes. We closely follow [2]. Let $\left(\Omega, \mathbb{B}, \mathcal{F}_{t}, \mathbb{P}\right)$ be a standard probability space with complete filtration $\mathcal{F}_{t}$ and let $\left(x_{k}\right)_{k \in \mathbb{Z}_{+}}$be a Markov chain in the phase space $(X, x)$ with transition probability $P(x, A)$, where $x \in X, A \in X$. Let $\left(\theta_{k}\right)_{k \in \mathbb{Z}_{+}}$be a renewal process which is a sequence of independent and identically distributed (i.i.d.) random variables with a common distribution function $F(x):=\mathbb{P}\left\{w: \theta_{k}(w) \leq x\right\}$. The random variables $\left(\theta_{k}\right)_{k \in \mathbb{Z}_{+}}$can be interpreted as lifetimes (operating periods, holding times, renewal periods) of a certain system in a random environment. From the renewal process $\left(\theta_{k}\right)_{k \in \mathbb{Z}_{+}}$we can construct another renewal process $\left(\tau_{k}\right)_{k \in \mathbb{Z}_{+}}$defined by

$$
\tau_{k}:=\sum_{n=0}^{k} \theta_{n}
$$

The random variables $\tau_{k}$ are called renewal times (or jump times). The process

$$
v(t):=\sup \left\{k: \tau_{k} \leq t\right\}
$$

is called the counting process. 
Definition 2.1 (see $[1,4])$. A homogeneous two-dimensional Markov chain $\left(x_{n}, \theta_{n}\right)_{n \in \mathbb{Z}_{+}}$on the phase space $X \times \mathbb{R}_{+}$is called a Markov renewal process (MRP) if its transition probabilities are given by the semi-Markov kernel

$$
Q(x, A, t)=\mathbb{P}\left\{x_{n+1} \in A, \theta_{n+1} \leq t \mid x_{n}=x\right\}, \quad \forall x \in X, A \in \mathcal{X}, t \in \mathbb{R}_{+} .
$$

Definition 2.2. The process

$$
x(t):=x_{v(t)}
$$

is called a semi-Markov process.

The ergodic theorem for a Markov renewal process and a semi-Markov process respectively can be found in [3, page 195], [1, page 66], and [4, page 113].

Let $\left(x_{n}, \theta_{n}\right)_{n \in \mathbb{Z}_{+}}$be a Markov renewal process on the phase space $X \times \mathbb{R}_{+}$with the semiMarkov kernel $Q(x, A, t)$ defined in (2.3), and let $x(t):=x_{v(t)}$ be a semi-Markov process where the counting process $v(t)$ is defined in (2.2). Let $\rho(x)$ be a bounded continuous function on $X$ such that $\rho(x)>-1$. We define the geometric Markov renewal process (GMRP) $\left\{S_{t}\right\}_{t \in \mathbb{R}_{+}}$as a stochastic functional $S_{t}$ defined by

$$
S_{t}:=S_{0} \prod_{k=1}^{v(t)}\left(1+\rho\left(x_{k}\right)\right), \quad t \in \mathbb{R}_{+}
$$

where $S_{0}>0$ is the initial value of $S_{t}$. We call this process $\left(S_{t}\right)_{t \in \mathbb{R}_{+}}$a geometric Markov renewal process by analogy with the geometric compound Poisson processes

$$
S_{t}^{*}=S_{0}^{*} \prod_{k=1}^{N(t)}\left(1+Y_{k}\right)
$$

where $S_{0}^{*}>0, N(t)$ is a standard Poisson process, $\left(Y_{k}\right)_{k \in Z_{+}}$are i.i.d. random variables. The geometric compound Poisson processes $\left\{S_{t}^{*}\right\}_{t \in \mathbb{R}_{+}}$in (2.6) is a trading model in many financial applications as a pure jump model $[5,6]$. The geometric Markov renewal processes $\left\{S_{t}\right\}_{t \in \mathbb{R}_{+}}$ in (2.5) will be our main trading model in further analysis.

Jump semi-Markov random evolutions, infinitesimal operators, and Martingale property of the GMRP were presented in [2]. For the convenience of readers we repeat them again in the following.

\subsection{Jump Semi-Markov Random Evolutions}

Let $C_{0}\left(\mathbb{R}_{+}\right)$be the space of continuous functions on $\mathbb{R}_{+}$vanishing at infinity, and let us define a family of bounded contracting operators $D(x)$ on $C_{0}\left(\mathbb{R}_{+}\right)$as follows:

$$
D(x) f(s):=f\left(s(1+\rho(x)), \quad x \in X, s \in \mathbb{R}_{+} .\right.
$$


With these contraction operators $D(x)$ we define the following jump semi-Markov random evolution (JSMRE) $V(t)$ of the geometric Markov renewal processes $\left\{S_{t}\right\}_{t \in \mathbb{R}_{+}}$in (2.5):

$$
V(t)=\prod_{k=1}^{v(t)} D\left(x_{k}\right):=D\left(x_{v(t)}\right) \circ D\left(x_{v(t)-1}\right) \circ \cdots \circ D\left(x_{1}\right)
$$

Using (2.7) we obtain from (2.8)

$$
V(t) f(s)=\prod_{k=1}^{v(t)} D\left(x_{k}\right) f(s)=f\left(s \prod_{k=1}^{v(t)}\left(1+\rho\left(x_{k}\right)\right)\right)=f\left(S_{t}\right),
$$

where $S_{t}$ is defined in (2.5) and $S_{0}=s$. Let $Q(x, A, t)$ be a semi-Markov kernel for Markov renewal process $\left(x_{n} ; \theta_{n}\right)_{n \in Z_{+}}$, that is, $Q(x, A, t)=P(x, A) G_{x}(t)$, where $P(x, A)$ is the transition probability of the Markov chain $\left(x_{n}\right)_{n \in \mathbb{Z}_{+}}$and $G_{x}(t)$ is defined by $G_{x}(t):=\mathbb{P}\left(\theta_{n+1} \leq t \mid x_{n}=x\right)$. Let

$$
u(t, x):=E_{x}[V(t) g(x(t))]:=E[V(t) g(x(t)) \mid x(0)=x]
$$

be the mean value of the semi-Markov random evolution $V(t)$ in (2.9).

The following theorem is proved in [1, page 60] and [4, page 38].

Theorem 2.3. The mean value $u(t, x)$ in $(2.10)$ of the semi-Markov random evolution $V(t)$ given by the solution of the following Markov renewal equation (MRE):

$$
u(t, x)-\int_{0}^{t} \int_{X} Q(x, d y, d s) D(y) u(t-s, y)=\bar{G}_{x}(t) g(x),
$$

where $\bar{G}_{x}(t)=1-G_{x}(t), G_{x}(t):=\mathbb{P}\left(\theta_{n+1} \leq t \mid x_{n}=x\right), g(x)$ is a bounded and continuous function on $X$.

\subsection{Infinitesimal Operators of the GMRP}

Let

$$
\begin{gathered}
1 \rho_{T}(x):=\frac{\rho(x)}{T}, \quad T>0 \\
S_{t}^{T}:=S_{0} \prod_{k=1}^{v(t T)}\left(1+\rho_{T}\left(x_{k}\right)\right)=S_{0} \prod_{k=1}^{v(t T)}\left(1+T^{-1} \rho\left(x_{k}\right)\right) .
\end{gathered}
$$

A detailed information about $\rho_{T}(x)$ and $S_{t}^{T}$ can be found in Section 4 of [2]. It can be easily shown that

$$
\ln \frac{S_{t}^{T}}{S_{0}}=\sum_{k=1}^{v(t T)} \ln \left(1+\frac{\rho\left(x_{k}\right)}{T}\right) .
$$


To describe martingale properties of the GMRP $\left(S_{t}\right)_{t \in \mathbb{R}_{+}}$in (2.5) we need to find an infinitesimal operator of the process

$$
\eta(t):=\sum_{k=1}^{v(t)} \ln \left(1+\rho\left(x_{k}\right)\right) .
$$

Let $\gamma(t):=t-\tau_{v(t)}$ and consider the process $(x(t), \gamma(t))$ on $X \times R_{+}$. It is a Markov process with infinitesimal operator

$$
\widehat{Q} f(x, t):=\frac{d f}{d t}+\frac{g_{x}(t)}{\bar{G}_{x}(t)} \int_{X}[P(x, d y) f(y, 0)-f(x, t)]
$$

where $g_{x}(t):=d G_{x}(t) / d t, \bar{G}_{x}(t)=1-G_{x}(t)$, where $f(x, t) \in C\left(X \times R_{+}\right)$. The infinitesimal operator for the process $\ln S(t)$ has the form:

$$
\widehat{A} f(z, x)=\frac{g_{x}(t)}{\bar{G}_{x}(t)} \int_{X} P(x, d y)[f(z+\ln (1+\rho(y), x)-f(z, x)]
$$

where $z:=\ln S_{0}$. The process $(\ln S(t), x(t), \gamma(t))$ is a Markov process on $R_{+} \times X \times R_{+}$with the infinitesimal operator

$$
\widehat{L} f(z, x, t)=\widehat{A} f(z, x, t)+\widehat{Q} f(z, x, t),
$$

where the operators $\widehat{A}$ and $\widehat{Q}$ are defined in (2.17) and (2.18), respectively. Thus we obtain that the process

$$
\widehat{m}(t):=f(\ln S(t), x(t), \gamma(t))-f(z, x, 0)-\int_{0}^{t}(\widehat{A}+\widehat{Q}) f(\ln S(u), x(u), \gamma(u)) d u
$$

is an $\widehat{\mathscr{f}}_{t}$-martingale, where $\hat{\mathscr{f}}_{t}:=\sigma(x(s), \gamma(s) ; 0 \leq s \leq t)$. If $x(t):=x_{v(t)}$ is a Markov process with kernel

$$
Q(x, A, t)=P(x, A)\left(1-e^{-\lambda(x) t}\right)
$$

namely, $G_{x}(t)=1-e^{-\lambda(x) t}$, then $g_{x}(t)=\lambda(x) e^{-\lambda(x) t}, \widehat{G}_{x}(t)=e^{-\lambda(x) t}, g_{x}(t) / \widehat{G}_{x}(t)=\lambda(x)$, and the operator $\widehat{A}$ in (2.17) has the form:

$$
\widehat{A} f(z)=\lambda(x) \int_{X} P(x, d y)[f(z+\ln (1+\rho(y)))-f(z)] .
$$

The process $(\ln S(t), x(t))$ on $R_{+} \times \mathrm{X}$ is a Markov process with infinitesimal operator

$$
\widehat{L} f(z, x)=\widehat{A} f(z, x)+Q f(z, x),
$$


where

$$
Q f(z, x)=\lambda(x) \int_{X} P(x, d y)(f(y)-f(x)) .
$$

It follows that the process

$$
m(t):=f(\ln S(t), x(t))-f(z, x)-\int_{0}^{t}(\widehat{A}+Q) f(\ln S(u), x(u)) d u
$$

is an $\mathcal{F}_{t}$-martingale, where $\mathcal{F}_{t}:=\sigma(x(u) ; 0 \leq u \leq t)$.

\subsection{Martingale Property of the GMRP}

Consider the geometric Markov renewal processes $\left(S_{t}\right)_{t \in \mathbb{R}_{+}}$

$$
S_{t}=S_{0} \prod_{k=1}^{v(t)}\left(1+\rho\left(x_{k}\right)\right)
$$

For $t \in[0, T]$ let us define

$$
L_{t}:=L_{0} \prod_{k=1}^{v(t)} h\left(x_{k}\right), \quad E L_{0}=1
$$

where $h(x)$ is a bounded continuous function such that

$$
\int_{X} h(y) P(x, d y)=1, \quad \int_{X} h(y) P(x, d y) p(y)=0 .
$$

If $E L_{T}=1$, then geometric Markov renewal process $S_{t}$ in $(2.25)$ is an $\left(\mathcal{F}_{t}, P^{*}\right)$-martingale, where measure $P^{*}$ is defined as follows:

$$
\begin{gathered}
\frac{d P^{*}}{d P}=L_{T}, \\
\mathcal{F}_{t}:=\sigma(x(s) ; 0 \leq s \leq t) .
\end{gathered}
$$

In the discrete case we have

$$
S_{n}=S_{0} \prod_{k=1}^{n}\left(1+\rho\left(x_{k}\right)\right)
$$

Let $L_{n}:=L_{0} \prod_{k=1}^{n} h\left(x_{k}\right), E L_{0}=1$, where $h(x)$ is defined in (2.27). If $E L_{N}=1$, then $S_{n}$ is an $\left(\mathcal{F}_{t}, P^{*}\right)$-martingale, where $d P^{*} / d P=L_{N}$, and $\mathcal{F}_{n}:=\sigma\left(x_{k} ; 0 \leq k \leq n\right)$. 


\section{Diffusion Approximation of the Geometric Markov Renewal Process (GMRP)}

Under an additional balance condition, averaging effect leads to diffusion approximation of the geometric Markov renewal process (GMRP). In fact, we consider the counting process $v(t)$ in (2.5) in the new accelerated scale of time $t T^{2}$, that is, $v \equiv v\left(t T^{2}\right)$. Due to more rapid changes of states of the system under the balance condition, the fluctuations are described by a diffusion processes.

\subsection{Ergodic Diffusion Approximation}

Let us suppose that balance condition is fulfilled for functional $S_{t}^{T}=S_{0} \prod_{k=1}^{v(t T)}\left(1+\rho_{T}\left(x_{k}\right)\right)$ :

$$
\widehat{\rho}=\frac{\int_{X} p(d x) \int_{X} P(x, d y) \rho(y)}{m}=0,
$$

where $p(x)$ is ergodic distribution of Markov chain $\left(x_{k}\right)_{k \in Z_{+}}$. Then $\widehat{S}(t)=S_{0}$, for all $t \in R^{+}$. Consider $S_{t}^{T}$ in the new scale of time $t T^{2}$ :

$$
S_{T}(t):=S_{t T^{2}}^{T}=S_{0} \prod_{k=1}^{v\left(t T^{2}\right)}\left(1+T^{-1} \rho\left(x_{k}\right)\right) .
$$

Due to more rapid jumps of $v\left(t T^{2}\right)$ the process $S_{T}(t)$ will be fluctuated near the point $S_{0}$ as $T \rightarrow+\infty$. By similar arguments similar to (4.3)-(4.5) in [2], we obtain the following expression:

$$
\ln \frac{S_{T}(t)}{S_{0}}=T^{-1} \sum_{k=1}^{v\left(t T^{2}\right)} \rho\left(x_{k}\right)-\frac{1}{2} T^{-2} \sum_{k=1}^{v\left(t T^{2}\right)} \rho^{2}\left(x_{k}\right)+T^{-2} \sum_{k=1}^{v\left(t T^{2}\right)} r\left(T^{-1} \rho\left(x_{k}\right)\right) \rho^{2}\left(x_{k}\right) .
$$

Algorithms of ergodic averaging give the limit result for the second term in (3.3) (see [1, page 43] and [4, page 88]):

$$
\lim _{T \rightarrow+\infty} \frac{1}{2} T^{-2} \sum_{k=1}^{v\left(t T^{2}\right)} \rho^{2}\left(x_{k}\right)=\frac{1}{2} t \widehat{\rho}_{2}
$$

where $\widehat{\rho}_{2}:=\int_{X} p(d x) \int_{X} P(x, d y) \rho^{2}(y) / m$. Using algorithms of diffusion approximation with respect to the first term in (3.3) we obtain [4, page 88]:

$$
\lim _{T \rightarrow+\infty} T^{-1} \sum_{k=1}^{v\left(t T^{2}\right)} \rho\left(x_{k}\right)=\sigma_{\rho} w(t)
$$

where $\sigma_{\rho}^{2}:=\int_{X} p(d x)\left[1 / 2 \int_{X} P(x, d y) \rho^{2}(y)+\int_{X} P(x, d y) \rho(y) R_{0} P(x, d y) \rho(y)\right] / m, R_{0}$ is a potential [3, page 68], of $\left(x_{n}\right)_{n \in Z_{+}}, w(t)$ is a standard Wiener process. The last term in (3.3) 
goes to zero as $T \rightarrow+\infty$. Let $\widehat{S}(t)$ be the limiting process for $S_{T}(t)$ in (3.3) as $T \rightarrow+\infty$. Taking limit on both sides of (3.3) we obtain

$$
\lim _{T \rightarrow+\infty} \ln \frac{S_{T}(t)}{S_{0}}=\ln \frac{\widehat{S}(t)}{S_{0}}=\sigma_{\rho} w(t)-\frac{1}{2} t \widehat{\rho}_{2}
$$

where $\sigma_{\rho}^{2}$ and $\hat{\rho}_{2}$ are defined in (3.4) and (3.5), respectively. From (3.6) we obtain

$$
\widehat{S}(t)=S_{0} e^{\sigma_{\rho} w(t)-(1 / 2) t \hat{\rho}_{2}}=S_{0} e^{-(1 / 2) t \hat{\rho}_{2}} e^{\sigma_{\rho} w(t)} .
$$

Thus, $\widehat{S}(t)$ satisfies the following stochastic differential equation (SDE):

$$
d \widehat{S}(t)=\widehat{S}(t)\left[\frac{1}{2}\left(\sigma_{\rho}^{2}-\hat{\rho}_{2}\right) d t+\sigma_{\rho} d w(t)\right]
$$

In this way we have the following corollary.

Corollary 3.1. The ergodic diffusion GMRP has the form

$$
\widehat{S}(t)=S_{0} e^{-(1 / 2) t \widehat{\rho}_{2}} e^{\sigma_{\rho} w(t)},
$$

and it satisfies the following SDE:

$$
\frac{d \widehat{S}(t)}{\widehat{S}(t)}=\frac{1}{2}\left(\sigma_{\rho}-\widehat{\rho}_{2}\right) d t+\sigma_{\rho} d w(t) .
$$

\subsection{Merged Diffusion Approximation}

Let us suppose that the balance condition satisfies the following:

$$
\hat{\rho}(k)=\frac{\int_{X_{k}} p_{k}(d x) \int_{X_{k}} P(x, d y) \rho(y)}{m(k)}=0,
$$

for all $k=1,2, \ldots, r$ where $\left(x_{n}\right)_{n \in Z_{+}}$is the supporting embedded Markov chain, $p_{k}$ is the stationary density for the ergodic component $X_{k}, m(k)$ is defined in [2], and conditions of reducibility of $X$ are fulfilled. Using the algorithms of merged averaging $[1,3,4]$ we obtain from the second part of the right hand side in (3.3):

$$
\lim _{T \rightarrow+\infty} \frac{1}{2} T^{-1} \sum_{k=1}^{v\left(t T^{2}\right)} \rho^{2}\left(x_{k}\right)=\frac{1}{2} \int_{0}^{t} \widehat{\rho}_{2}(\widehat{x}(s)) d s,
$$


where

$$
\widehat{\rho}_{2}(k):=\frac{\int_{X_{k}} p_{k}(d x) \int_{X_{k}} P(x, d y) \rho^{2}(y)}{m(k)}
$$

using the algorithm of merged diffusion approximation that $[1,3,4]$ obtain from the first part of the right hand side in (3.3):

$$
\lim _{T \rightarrow+\infty} T^{-1} \sum_{k=1}^{v\left(t T^{2}\right)} \rho\left(x_{k}\right)=\int_{0}^{t} \widehat{\sigma}_{\rho}(\widehat{x}(s)) d w(s),
$$

where

$$
\widehat{\sigma}_{\rho}^{2}(k):=\int_{X_{k}} p_{k}(d x) \int_{X_{k}} P(x, d y) \rho^{2}(y)+\int_{X_{k}} P(x, d y) \rho(y) R_{0} \int_{X_{k}} \frac{P(x, d y) \rho(y)}{m(k)} .
$$

The third term in (3.3) goes to 0 as $T \rightarrow+\infty$. In this way, from (3.3) we obtain:

$$
\lim _{T \rightarrow+\infty} \ln \frac{S_{T}(t)}{S_{0}}=\ln \frac{\tilde{S}(t)}{S_{0}}=\int_{0}^{t} \widehat{\sigma}_{\rho}(\widehat{x}(s)) d w(s)-\frac{1}{2} \int_{0}^{t} \widehat{\rho}_{2}(\widehat{x}(s)) d s,
$$

where $\widetilde{S}(t)$ is the limit $S_{T}(t)$ as $T \rightarrow+\infty$. From (3.16) we obtain

$$
\widetilde{S}(t)=S_{0} e^{-(1 / 2) \int_{0}^{t} \hat{\rho}^{2}(\widehat{x}(s)) d s+\int_{0}^{t} \widehat{\sigma}_{\rho}(\widehat{x}(s)) d w(s)} .
$$

Stochastic differential equation (SDE) for $\breve{S}(t)$ has the following form:

$$
\frac{d \widetilde{S}(t)}{\widetilde{S}(t)}=\frac{1}{2}\left(\widehat{\sigma}_{\rho}^{2}(\widehat{x}(t))-\widehat{\rho}_{2}(\widehat{x}(t))\right) d t+\widehat{\sigma}_{\rho}(\widehat{x}(t)) d w(t),
$$

where $\widehat{x}(t)$ is a merged Markov process.

In this way we have the following corollary.

Corollary 3.2. Merged diffusion GMRP has the form (3.17) and satisfies the SDE (3.18).

\subsection{Diffusion Approximation under Double Averaging}

Let us suppose that the phase space $\widehat{X}=\{1,2, \ldots, r\}$ of the merged Markov process $\widehat{x}(t)$ consists of one ergodic class with stationary distributions $\left(\widehat{p}_{k} ; k=\{1,2, \ldots r\}\right)$. Let us also suppose that the balance condition is fulfilled:

$$
\sum_{k=1}^{r} \widehat{p}_{k} \widehat{\rho}(k)=0
$$


Then using the algorithms of diffusion approximation under double averaging (see $[3$, page 188], [1, page 49] and [4, page 93]) we obtain:

$$
\lim _{T \rightarrow+\infty} \ln \frac{S_{T}(t)}{S_{0}}=\ln \frac{\breve{S}(t)}{S_{0}}=\check{\sigma}_{\rho} w(t)-\frac{1}{2} \check{p}_{2} t,
$$

where

$$
\breve{\sigma}_{\rho}^{2}:=\sum_{k=1}^{r} \widehat{p}_{k} \widehat{\sigma}_{\rho}^{2}(k), \quad \check{p}_{2}:=\sum_{k=1}^{r} \widehat{p}_{k} \widehat{\rho}_{2}(k),
$$

and $\hat{\rho}_{2}(k)$ and $\widehat{\sigma}_{\rho}^{2}(k)$ are defined in (3.13) and (3.15), respectively. Thus, we obtain from (3.20):

$$
\breve{S}(t)=S_{0} e^{-(1 / 2) \check{\check{r}}_{2} t+\check{\sigma}_{\rho} w(t)} .
$$

Corollary 3.3. The diffusion GMRP under double averaging has the form

$$
\breve{S}(t)=S_{0} e^{-(1 / 2) \check{\rho}_{2} t+\check{\sigma}_{\rho} w(t)},
$$

and satisfies the $S D E$

$$
\frac{d \check{S}(t)}{\breve{S}(t)}=\frac{1}{2}\left(\breve{\sigma}_{\rho}^{2}-\check{\rho}_{2}\right) d t+\check{\sigma}_{\rho} d w(t)
$$

\section{Proofs}

In this section we present proofs of results in Section 3. All the above-mentioned results are obtained from the general results for semi-Markov random evolutions $[3,4]$ in series scheme. The main steps of proof are (1) weak convergence of $S_{t}^{T}$ in Skorokhod space $D_{R}[0,+\infty)[7$, page 148]; (2) solution of martingale problem for the limit process $\widehat{S}(t)$; (3) characterization of the limit measure for the limit process $\widehat{S}(t) ;(4)$ uniqueness of solution of martingale problem. We also give here the rate of convergence in the diffusion approximation scheme.

\subsection{Diffusion Approximation (DA)}

Let

$$
G_{t}^{T}:=T^{-1} \sum_{k=0}^{v\left(t T^{2}\right)} \rho\left(x_{k}\right), \quad G_{n}^{T}:=G_{\tau_{n} T^{-1}}^{T}, \quad G_{0}^{T}=\ln s,
$$

and the balance condition is satisfied:

$$
\widehat{\rho}:=\int_{X} p(d x) \int_{X} P(x, d y) \rho(y)=0 .
$$


International Journal of Stochastic Analysis

Let us define the functions

$$
\phi^{T}(s, x):=f(s)+T^{-1} \phi_{f}^{1}(s, x)+T^{-2} \phi_{f}^{2}(s, x),
$$

where $\phi_{f}^{1}$ and $\phi_{f}^{2}$ are defined as follows:

$$
\begin{gathered}
(P-I) \phi_{f}^{1}(s, x)=\rho(x) f(s), \\
(P-I) \phi_{f}^{2}(s, x)=[-A(x)+\widehat{A}] f(s),
\end{gathered}
$$

where

$$
\widehat{A}:=\int_{X} p(d x) A(x)
$$

and $A(x):=\left[\rho^{2}(x) / 2+\rho(x)\left(R_{0}-I\right) \rho(x)\right] d^{2} / d s^{2}$. From the balance condition (4.2) and equality $\Pi(\widehat{A}-A(x))=0$ it follows that both equations in (4.3) simultaneously solvable and the solutions $\phi_{f}^{i}(s, x)$ are bounded functions, $i=1,2$.

We note that

$$
f\left(S_{n+1}^{T}\right)-f\left(G_{n}^{T}\right)=\frac{1}{T} \rho\left(x_{n}\right) \frac{d f\left(x_{n}\right)}{d s}
$$

and define

$$
\phi^{T}(s, x):=f(s)+T^{-1} \phi_{f}^{1}(s, x)+T^{-2} \phi_{f}^{2}(s, x)
$$

where $\phi_{f}^{1}(s, x)$ and $\phi_{f}^{2}(s, x)$ are defined in (4.4) and (4.5), respectively. We note, that $G_{n+1}^{T}-$ $G_{n}^{T}=T^{-1} \rho\left(x_{n}\right)$.

\subsection{Martingale Problem for the Limiting Problem $G_{0}(t)$ in DA}

Let us introduce the family of functions:

$$
\begin{aligned}
\psi^{T}(s, t):= & \phi^{T}\left(G_{\left[t T^{2}\right]}^{T}, x_{\left[t T^{2}\right]}\right)-\phi^{T}\left(G_{\left[s t^{2}\right]}^{T}, x_{\left[s T^{2}\right]}\right) \\
& -\sum_{j=\left[s T^{2}\right]}^{\left[t T^{2}\right]-1} E\left[\phi^{T}\left(G_{j}^{T}, x_{j+1}\right)-\phi^{T}\left(G_{j}^{T}, x_{j}\right) \mid \mathcal{F}_{j}\right],
\end{aligned}
$$

where $\phi^{T}$ are defined in (4.7) and $G_{j}^{T}$ is defined by

$$
G_{\tau_{n} / T}^{T}=\frac{1}{T} \sum_{k=0}^{n} \rho\left(x_{k}\right)
$$


Functions $\psi^{T}(s, t)$ are $\mathcal{F}_{\left[t T^{2}\right]}$-martingale by $t$. Taking into account the expression (4.6) and (4.7), we find the following expression:

$$
\begin{aligned}
& \psi^{T}(s, t)=f\left(G_{\left[t T^{2}\right]}^{T}\right)-f\left(G_{\left[s T^{2}\right]}^{T}\right)+\epsilon\left[\phi_{f}^{1}\left(G_{\left[t T^{2}\right]^{\prime}}^{T} x_{\left[t T^{2}\right]}\right)-\phi_{f}^{1}\left(G_{\left[s t^{2}\right]}^{T}, x_{\left[s T^{2}\right]}\right)\right] \\
& +\epsilon^{2}\left[\phi_{f}^{2}\left(G_{\left[t T^{2}\right]}^{T}, x_{\left[t T^{2}\right]}\right)-\phi_{f}^{2}\left(G_{\left[s T^{2}\right]^{T}}^{T}, x_{\left[s T^{2}\right]}\right)\right] \\
& -T^{-1} \sum_{j=\left[s T^{2}\right]}^{\left[t T^{2}\right]-1}\left\{\rho\left(x_{j}\right) \frac{d f\left(G_{j}^{T}\right)}{d g}+E\left(\phi_{f}^{1}\left(G_{j}^{T}, x_{j+1}\right)-\phi_{f}^{2}\left(G_{j}^{T}, x_{j}\right) \mid \mathcal{F}_{j}\right)\right\} \\
& -T^{-2} \sum_{j=\left[s T^{2}\right]}^{\left[t T^{2}\right]-1}\left\{2^{-1} \rho^{2}\left(x_{j}\right) \frac{d f\left(G_{j}^{T}\right)}{d g}+\rho\left(x_{j}\right) E\left(\frac{d \phi_{f}^{1}\left(G_{j}^{T}, x_{j+1}\right)}{d g} \mid \mathcal{F}_{j}\right)\right. \\
& \left.+E\left[\phi_{f}^{2}\left(G_{j}^{T}, x_{j+1}\right)-\phi_{f}^{2}\left(G_{j}^{T}, x_{j}\right) \mid \mathscr{F}_{j}\right]\right\}+o\left(T^{-2}\right) \\
& =f\left(G_{\left[t T^{2}\right]}^{T}\right)-f\left(G_{\left[s T^{2}\right]}^{T}\right)+\left[\phi_{f}^{1}\left(G_{\left[t T^{2}\right]}^{T}, x_{\left[t T^{2}\right]}\right)-\phi_{f}^{1}\left(G_{\left[s T^{2}\right]^{\prime}}^{T} x_{\left[s T^{2}\right]}\right)\right] \\
& +T^{-2}\left[\phi_{f}^{2}\left(G_{\left[t T^{2}\right]}^{T}, x_{\left[t T^{2}\right]}\right)-\phi_{f}^{2}\left(G_{\left[s T^{2}\right]}^{T}, x_{\left[s T^{2}\right]}\right)\right]-T^{-2} \sum_{j=\left[s T^{2}\right]}^{\left[t T^{2}\right]-1} \widehat{A} f\left(G_{j}^{T}\right)+O\left(T^{-2}\right),
\end{aligned}
$$

where $O\left(T^{-2}\right)$ is the sum of terms with $T^{-2}$ nd order. Since $\psi^{T}(0, t)$ is $\mathcal{F}_{\left[t T^{2}\right]}$-martingale with respect to measure $Q_{T}$, generated by process $G_{T}(t)$ in (4.1), then for every scalar linear continuous functional $\eta_{0}^{s}$ we have from (4.8)-(4.10):

$$
\begin{aligned}
0= & E^{T}\left[\left(\psi^{T}(s, t) \eta_{0}^{s}\right]\right. \\
= & E^{T}\left[\left(f\left(G_{\left[t T^{2}\right]}^{T}\right)-f\left(G_{\left[s T^{2}\right]}^{T}\right)-T^{-2} \sum_{j=\left[s T^{2}\right]}^{\left[t T^{2}\right]-1} \widehat{A} f\left(G_{j}^{T}\right)\right) \eta_{0}^{s}\right] \\
& -T^{-1} E^{T}\left[\left(\phi_{f}^{1}\left(G_{\left[t T^{2}\right]}^{T}, x_{\left[t T^{2}\right]}\right)-\phi_{f}^{1}\left(G_{\left[s T^{2}\right]^{\prime}}^{T} x_{\left[s T^{2}\right]}\right)\right) \eta_{0}^{s}\right] \\
& -T^{-2} E^{T}\left[\left(\phi_{f}^{2}\left(G_{\left[t T^{2}\right]}^{T}, x_{\left[t T^{2}\right]}\right)-\phi_{f}^{2}\left(G_{\left[s T^{2}\right]}^{T}, x_{\left[s T^{2}\right]}\right)\right) \eta_{0}^{s}\right]-O\left(T^{-2}\right),
\end{aligned}
$$


where $E^{T}$ is a mean value by measure $Q_{T}$. If the process $G_{\left[t T^{2}\right]}^{T}$ converges weakly to some process $G_{0}(t)$ as $T \rightarrow+\infty$, then from (4.11) we obtain

$$
0=E^{T}\left[\left(f\left(G_{0}(t)\right)-f\left(G_{0}(s)\right)-\int_{s}^{t} \widehat{A} f\left(G_{0}(u)\right) d u\right]\right.
$$

that is, the process

$$
f\left(G_{0}(t)\right)-f\left(G_{0}(s)\right)-\int_{s}^{t} \widehat{A} f\left(G_{0}(u)\right) d u
$$

is a continuous $Q_{T}$-martingale. Since $\widehat{A}$ is the second order differential operator and coefficient $\sigma_{1}^{2}$ is positively defined, where

$$
\sigma_{1}^{2}:=\int_{X} \pi(d x)\left[\frac{\rho^{2}(x)}{2}+\rho(x) R_{0} \rho(x)\right]
$$

then the process $G_{0}(t)$ is a Wiener process with variance $\sigma_{1}^{2}$ in (4.14): $G_{0}(t)=\sigma w(t)$. Taking into account the renewal theorem for $v(t)$, namely, $T^{-1} v\left(t T^{2}\right) \rightarrow_{T \rightarrow+\infty} t / m$, and the following representation

$$
G_{T}(t)=T^{-1} \sum_{k=0}^{v\left(t T^{2}\right)} \rho\left(x_{k}\right)=T^{-1} \sum_{k=0}^{\left[t T^{2}\right]} \rho\left(x_{k}\right)+T^{-1} \sum_{k=\left[t T^{2}\right]+1}^{v\left(t T^{2}\right)} \rho\left(x_{k}\right)
$$

we obtain, replacing $\left[t T^{2}\right]$ by $v\left(t T^{2}\right)$, that process $G_{T}(t)$ converges weakly to the process $\widehat{G}_{0}(t)$ as $T \rightarrow+\infty$, which is the solution of such martingale problem:

$$
f\left(\widehat{G}_{0}(t)\right)-f\left(\widehat{G}_{0}(s)\right)-\int_{s}^{t} \widehat{A}_{0} f\left(\widehat{G}_{0}(u)\right) d u
$$

is a continuous $Q_{T}$-martingale, where $\widehat{A}_{0}:=\widehat{A} / m$, and $\widehat{A}$ is defined in (4.5)-(4.5).

\subsection{Weak Convergence of the Processes $G_{T}(t)$ in DA}

From the representation of the process $G_{T}(t)$ it follows that

$$
\begin{aligned}
\Delta_{T}(s, t): & =\left|G_{T}(t)-G_{T}(s)\right|=\left|T^{-1} \sum_{k=v\left(s T^{2}\right)+1}^{v\left(t T^{2}\right)} \rho\left(x_{k}\right)\right| \\
& \leq T^{-1} \sup _{x} \rho(x)\left|v\left(t T^{2}\right)-v\left(s T^{2}\right)-1\right| .
\end{aligned}
$$


This representation gives the following estimation:

$$
\left|\Delta_{T}\left(t_{1}, t_{2}\right)\right|\left|\Delta_{T}\left(t_{2}, t_{3}\right)\right| \leq T^{-2}\left(\sup _{x} \rho(x)\right)^{2}\left|v\left(t_{3} T^{2}\right)-v\left(t_{1} T^{2}\right)\right|^{2}
$$

Taking into account the same reasonings as in [2] we obtain the weak convergence of the processes $G_{T}(t)$ in DA.

\subsection{Characterization of the Limiting Measure $Q$ for $Q_{T}$ as $T \rightarrow+\infty$ in DA}

From Section 4.3 (see also Section 4.1.4 of [2]) it follows that there exists a sequence $T_{n}$ such that measures $Q_{T_{n}}$ converge weakly to some measure $Q$ on $D_{R}[0,+\infty)$ as $T \rightarrow+\infty$, where $D_{R}[0,+\infty)$ is the Skorokhod space [7, page 148]. This measure is the solution of such martingale problem: the following process

$$
m(s, t):=f\left(\widehat{G}_{0}(t)\right)-f\left(\widehat{G}_{0}(s)\right)-\int_{s}^{t} \widehat{A}_{0} f\left(\widehat{G}_{0}(u)\right) d u
$$

is a $Q$-martingale for all $f(g) \in C^{2}(R)$ and

$$
\operatorname{Em}(s, t) \eta_{0}^{s}=0
$$

for scalar continuous bounded functional $\eta_{0}^{s}, E$ is a mean value by measure $Q$. From (4.19) it follows that $E^{T} m^{T}(s, t) \eta_{0}^{s}=0$, and it is necessary to show that the limiting passing in (4.1) goes to the process in (3.12) as $T \rightarrow+\infty$. From equality (4.11) we find that $\lim _{T_{n} \rightarrow+\infty} E^{T_{n}} m(s, t) \eta_{0}^{s}=$ $\operatorname{Em}(s, t) \eta_{0}^{s}$. Moreover, from the following expression

$$
\left|E^{T} m(s, t) \eta_{0}^{s}-E m(s, t) \eta_{0}^{s}\right| \leq\left|\left(E^{T}-E\right) m(s, t) \eta_{0}^{s}\right|+E^{T}\left|m(s, t)-m^{T}(s, t)\right|\left|\eta_{0}^{s}\right| \longrightarrow_{T \rightarrow+\infty} 0,
$$

we obtain that there exists the measure $Q$ on $D_{R}[0,+\infty)$ which solves the martingale problem for the operator $\widehat{A}_{0}$ (or, equivalently, for the process $\widehat{G}_{0}(t)$ in the form (4.12)). Uniqueness of the solution of the martingale problem follows from the fact that operator $\widehat{A}_{0}$ generates the unique semigroup with respects to the Wiener process with variance $\sigma_{1}^{2}$ in (4.14). As long as the semigroup is unique then the limit process $\widehat{G}_{0}(t)$ is unique. See [3, Chapter 1$]$.

\subsection{Calculation of the Quadratic Variation for GMRP}

If $G_{n}^{T}=G_{T^{-1} \tau_{n}}^{T}$, the sequence

$$
m_{n}^{T}:=G_{n}^{T}-G_{0}^{T}-\sum_{k=0}^{n-1} E\left[G_{k+1}^{T}-G_{k}^{T} \mid \mathscr{F}_{k}\right], \quad G_{0}^{T}=g
$$


is $\mathcal{F}_{n}$-martingale, where $\mathcal{F}_{n}:=\sigma\left\{x_{k}, \theta_{k} ; 0 \leq k \leq n\right\}$. From the definition it follows that the characteristic $\left\langle m_{n}^{T}\right\rangle$ of the martingale $m_{n}^{T}$ has the form

$$
\left\langle m_{n}^{T}\right\rangle=\sum_{k=0}^{n-1} E\left[\left(m_{k+1}^{T}-m_{k}^{T}\right)^{2} \mid \mathcal{F}_{k}\right]
$$

To calculate $\left\langle m_{n}^{T}\right\rangle$ let us represent $m_{n}^{T}$ in (4.22) in the form of martingale-difference:

$$
m_{n}^{T}=\sum_{k=0}^{n-1}\left[G_{k+1}^{T}-E\left(G_{k+1}^{T} \mid \mathscr{F}_{k}\right)\right]
$$

From representation

$$
G_{n+1}^{T}-G_{n}^{T}=\frac{1}{T} \rho\left(x_{n}\right)
$$

it follows that $E\left(G_{k+1}^{T} \mid \mathscr{F}_{k}\right)=G_{k}^{T}+T^{-1} \rho\left(x_{k}\right)$, that is why

$$
G_{k+1}^{T}-E\left(G_{k+1}^{T} \mid \mathcal{F}_{k}\right)=T^{-1}\left(\rho\left(x_{k}\right)-P \rho\left(x_{k}\right)\right) \text {. }
$$

Since from (4.22) it follows that

$$
m_{k+1}^{T}-m_{k}^{T}=G_{k+1}^{T}-E\left(G_{k+1}^{T} \mid \mathscr{F}_{k}\right)=T^{-1}\left(\rho\left(x_{k}\right)-P \rho\left(x_{k}\right)\right),
$$

then substituting (4.27) in (4.23) we obtain

$$
\left\langle m_{n}^{T}\right\rangle=T^{-2} \sum_{k=0}^{n-1}\left[(I-P) \rho\left(x_{k}\right)\right]^{2}
$$

In an averaging scheme (see [2]) for GMRP in the scale of time $t T$ we obtain that $\left\langle m_{[t T]}^{T}\right\rangle$ goes to zero as $T \rightarrow+\infty$ in probability, which follows from (4.27):

$$
\left\langle m_{[t T]}^{T}\right\rangle=T^{-2} \sum_{k=0}^{[t T]-1}\left[(I-P) \rho\left(x_{k}\right)\right]^{2} \longrightarrow 0 \quad \text { as } T \longrightarrow+\infty
$$

for all $t \in R_{+}$. In the diffusion approximation scheme for GMRP in scale of time $t T^{2}$ from (4.27) we obtain that characteristic $\left\langle m_{\left[t T^{2}\right]}^{T}\right\rangle$ does not go to zero as $T \rightarrow+\infty$ since

$$
\left\langle m_{\left[t T^{2}\right]}^{T}\right\rangle=T^{-2} \sum_{k=0}^{\left[t T^{2}\right]-1}\left[(I-P) \rho\left(x_{k}\right)\right]^{2} \longrightarrow t \sigma_{1}^{2},
$$

where $\sigma_{1}^{2}:=\int_{X} \pi(d x)[(I-P) \rho(x)]^{2}$. 


\subsection{Rates of Convergence for GMRP}

Consider the representation (4.22) for martingale $m_{n}^{T}$. It follows that

$$
G_{n}^{T}=g+m_{n}^{T}+\sum_{k=0}^{n-1} E\left[G_{k+1}^{T}-G_{k}^{T} \mid \mathscr{F}_{k}\right]
$$

In diffusion approximation scheme for GMRP the limit for the process $G_{\left[t T^{2}\right]}^{T}$ as $T \rightarrow+\infty$ will be diffusion process $\widehat{S}(t)$ (see (3.10)). If $m_{0}(t)$ is the limiting martingale for $m_{\left[t T^{2}\right]}^{T}$ in (4.22) as $T \rightarrow+\infty$, then from (4.31) and (3.10) we obtain

$$
E\left[G_{\left[t T^{2}\right]}^{T}-\widehat{S}(t)\right]=E\left[m_{\left[t T^{2}\right]}^{T}-m_{0}(t)\right]+T^{-1} \sum_{k=0}^{\left[t T^{2}\right]-1} \rho\left(x_{k}\right)-\widehat{S}(t) .
$$

Since $E\left[m_{\left[t T^{2}\right]}^{T}-m_{0}(t)\right]=0$, (because $m_{\left[t T^{2}\right]}^{T}$ and $m_{0}(t)$ are zero-mean martingales) then from (4.32) we obtain:

$$
\left|E\left[G_{\left[t T^{2}\right]}^{T}-\widehat{S}(t)\right]\right| \leq T^{-1}\left|\sum_{k=0}^{\left[t T^{2}\right]-1} \rho\left(x_{k}\right)-\widehat{S}(t) T\right|
$$

Taking into account the balance condition $\int_{X} \pi(d x) \rho(x)=0$ and the central limit theorem for a Markov chain [4, page 98], we obtain

$$
\left|\sum_{k=0}^{\left[t T^{2}\right]-1} \rho\left(x_{k}\right)-\widehat{S}(t) T\right|=C_{1}\left(t_{0}\right)
$$

where $C_{1}\left(t_{0}\right)$ is a constant depending on $t_{0}, t \in\left[0, t_{0}\right]$. From (4.33), (4.2), and (4.32) we obtain:

$$
\left|E\left[G_{\left[t T^{2}\right]}^{T}-\widehat{S}(t)\right]\right| \leq T^{-1} C_{1}\left(t_{0}\right) .
$$

Thus, the rates of convergence in diffusion scheme has the order $T^{-1}$.

\section{Merged Diffusion Geometric Markov Renewal Process in the Case of Two Ergodic Classes}

\subsection{Two Ergodic Classes}

Let $P(x, A):=\mathbb{P}\left\{x_{n+1} \in A \mid x_{n}=x\right\}$ be the transition probabilities of supporting embedded reducible Markov chain $\left\{x_{n}\right\}_{n \geq 0}$ in the phase space $X$. Let us have two ergodic classes $X_{0}$ and $X_{1}$ of the phase space such that:

$$
X=X_{0} \cup X_{1}, \quad X_{0} \cap X_{1}=\emptyset .
$$


Let $\{\hat{X}=\{0,1\}, \mho\}$ be the measurable merged phase space. A stochastic kernel $P_{0}(x, A)$ is consistent with the splitting (5.1) in the following way:

$$
P_{0}\left(x, X_{k}\right)=1_{k}:=\left\{\begin{array}{ll}
1, & x \in X_{k} \\
0, & x \notin X_{k},
\end{array} \quad k=0,1 .\right.
$$

Let the supporting embedded Markov chain $\left(x_{n}\right)_{n \in Z_{+}}$with the transition probabilities $\mathrm{P}_{0}(x, A)$ be uniformly ergodic in each class $X_{k}, k=0,1$ and have a stationary distribution $\pi_{k}(d x)$ in the classes $X_{k}, k=0,1$ :

$$
\pi_{k}(A)=\int_{X_{k}} \pi_{k}(d x) P_{0}(x, A), \quad A \subset X_{k}, k=0,1
$$

Let the stationary escape probabilities of the embedded Markov chain $\left(x_{n}\right)_{n \in Z_{+}}$with transition probabilities $P(x, A):=\mathbb{P}\left\{x_{n+1} \in A \mid x_{n}=x\right\}$ be positive and sufficiently small, that is,

$$
q_{k}(A)=\int_{X_{k}} \pi_{k}(d x) P\left(x, X \backslash X_{k}\right)>0, \quad k=0,1
$$

Let the stationary sojourn time in the classes of states be uniformly bounded, namely,

$$
0 \leq C_{1} \leq m_{k}:=\int_{X_{k}} \pi_{k}(d x) m(x) \leq C_{2}, \quad k=0,1,
$$

where

$$
m(x):=\int_{0}^{\infty} \bar{G}_{x}(t) d t
$$

\subsection{Algorithms of Phase Averaging with Two Ergodic Classes}

The merged Markov chain $\left(\widehat{x}_{n}\right)_{n \in Z_{+}}$in merged phase space $\hat{X}$ is given by matrix of transition probabilities

$$
\begin{gathered}
\widehat{P}=\left(\hat{p}_{k r}\right)_{k, r=0,1} ; \\
\widehat{p}_{01}=1-\widehat{p}_{11}=\int_{X_{1}} \pi_{1}(d x) P\left(x, X_{0}\right)=1-\int_{X_{1}} \pi_{1}(d x) P\left(x, X_{1}\right) ; \\
\hat{p}_{01}=1-\widehat{p}_{00}=\int_{X_{0}} \pi_{0}(d x) P\left(x, X_{1}\right)=1-\int_{X_{0}} \pi_{0}(d x) P\left(x, X_{0}\right) .
\end{gathered}
$$


As $\widehat{p}_{k r} \neq 0, k=0,1$, then $\widehat{x}_{n}$ has virtual transitions. Intensities $\widehat{\Lambda}_{k}$ of sojourn times $\widehat{\theta}_{k}, k=0,1$, of the merged MRP are calculated as follows:

$$
\widehat{\Lambda}_{k}=\frac{1}{m_{k}}, \quad m_{k}=\int_{X_{k}} \pi_{k}(d x) m(x), k=0,1
$$

And, finally, the merged MRP $\left(\widehat{x}_{n}, \widehat{\theta}\right)_{n \in Z_{+}}$in the merged phase space $\widehat{X}$ is given by the stochastic matrix

$$
\widehat{Q}(t)=\left(\widehat{Q}_{k r}\right)_{k, r=0,1}:=\widehat{p}_{k r}\left(1-e^{-\widehat{\Lambda}_{k} t}\right), \quad k, r=0,1 .
$$

Hence, the initial semi-Markov system is merged to a Markov system with two classes.

\subsection{Merged Diffusion Approximation in the Case of Two Ergodic Classes}

The merged diffusion GMRP in the case of two ergodic classes has the form:

$$
\widetilde{S}(t)=S_{0} e^{-(1 / 2) \int_{0}^{t} \widehat{\rho}^{2}(\widehat{x}(s)) d s+\int_{0}^{t} \widehat{\sigma}_{\rho}(\widehat{x}(s)) d w(s)}
$$

which satisfies the stochastic differential equation (SDE):

$$
\frac{d \widetilde{S}(t)}{\widetilde{S}(t)}=\frac{1}{2}\left(\widehat{\sigma}_{\rho}^{2}(\widehat{x}(t))-\widehat{\rho}^{2}(\widehat{x}(t))\right) d t+\widehat{\sigma}_{\rho}(\widehat{x}(t)) d w(t),
$$

where

$$
\begin{gathered}
\hat{\rho}^{2}(1):=\int_{X_{1}} p_{1}(d x) \int_{X_{1}} \frac{P(x, d y) \rho^{2}(y)}{m(1)} \\
\hat{\rho}^{2}(0):=\int_{X_{0}} p_{0}(d x) \int_{X_{0}} \frac{P(x, d y) \rho^{2}(y)}{m(0)} \\
\widehat{\sigma}_{\rho}^{2}(1):=\int_{X_{1}} p_{1}(d x) \int_{X_{1}} P(x, d y) \rho^{2}(y)+\int_{X_{1}} P(x, d y) \rho(y) R_{0} \int_{X_{1}} \frac{P(x, d y) \rho(y)}{m(1)}, \\
\widehat{\sigma}_{\rho}^{2}(0):=\int_{X_{0}} p_{0}(d x) \int_{X_{0}} P(x, d y) \rho^{2}(y)+\int_{X_{0}} P(x, d y) \rho(y) R_{0} \int_{X_{0}} \frac{P(x, d y) \rho(y)}{m(0)},
\end{gathered}
$$

$\widehat{x}(t)$ is a merged Markov process in $\widehat{X}=\{0,1\}$ with stochastic matrix $\widehat{Q}(t)$ in (5.9). 
International Journal of Stochastic Analysis

\section{European Call Option Pricing Formulas for Diffusion GMRP}

\subsection{Ergodic Geometric Markov Renewal Process}

As we have seen in Section 3, an ergodic diffusion GMRP $\widehat{S}(t)$ satisfies the following SDE (see (3.10)):

$$
\frac{d \widehat{S}(t)}{\widehat{S}(t)}=\frac{1}{2}\left(\sigma_{\rho}-\widehat{\rho}_{2}\right) d t+\sigma_{\rho} d w(t)
$$

where

$$
\begin{aligned}
\hat{\rho}_{2} & =\int_{X} p(d x) \int_{X} \frac{P(x, d y) \rho^{2}(y)}{m}, \\
\sigma_{\rho}^{2} & =\int_{X} p(d x)\left[\frac{1}{2} \int_{X} P(x, d y) \rho^{2}(y)+\int_{X} \frac{P(x, d y) \rho(y) R_{0} P(x, d y) \rho(y)}{m} .\right.
\end{aligned}
$$

The risk-neutral measure $P^{*}$ for the process in (6.1) is:

$$
\frac{d P^{*}}{P}=\exp \left\{-\theta t-\frac{1}{2} \theta^{2} w(t)\right\}
$$

where

$$
\theta=\frac{\left((1 / 2)\left(\sigma_{\rho}-\hat{\rho}_{2}\right)-r\right)}{\sigma_{\rho}}
$$

Under $P^{*}$, the process $e^{-r t} \widehat{S}_{t}$ is a martingale and the process $w^{*}(t)=w(t)+\theta t$ is a Brownian motion. In this way, in the risk-neutral world, the process $\widehat{S}_{t}$ has the following form

$$
\frac{d \widehat{S}(t)}{\widehat{S}(t)}=r d t+\sigma_{\rho} d w^{*}(t) .
$$

Using Black-Scholes formula (see [8]) we obtain the European call option pricing formula for our model (6.6):

$$
C=S_{0} \Phi\left(d_{+}\right)-K e^{-r T} \Phi\left(d_{-}\right)
$$

where

$$
\begin{aligned}
& d_{+}=\frac{\ln \left(S_{0} / K\right)+\left(r+(1 / 2) \sigma_{\rho} t\right)}{\sigma_{\rho} \sqrt{t}}, \\
& d_{-}=\frac{\ln \left(S_{0} / K\right)+\left(r-(1 / 2) \sigma_{\rho} t\right)}{\sigma_{\rho} \sqrt{t}},
\end{aligned}
$$

$\Phi(x)$ is a normal distribution and $\sigma_{\rho}$ is defined in (6.3). 


\subsection{Double Averaged Diffusion GMRP}

Using the similar arguments as in (6.1)-(6.7), we can get European call option pricing formula for a double averaged diffusion GMRP in (3.24):

$$
\frac{d \check{S}(t)}{\breve{S}(t)}=\frac{1}{2}\left(\breve{\sigma}_{\rho}^{2}-\check{\rho}_{2}\right) d t+\check{\sigma}_{\rho} d w(t)
$$

where $\breve{\sigma}_{\rho}^{2}$ and $\check{\rho}_{2}$ are defined in (3.21), (see also (3.13)), and (3.15). Namely, the European call option pricing formula for a double averaged diffusion GMRP is:

$$
C=S_{0} \Phi\left(d_{+}\right)-K e^{-r T} \Phi\left(d_{-}\right),
$$

where

$$
\begin{aligned}
& d_{+}=\frac{\ln \left(S_{0} / K\right)+\left(r+(1 / 2) \check{\sigma}_{\rho} t\right)}{\check{\sigma}_{\rho} \sqrt{t}}, \\
& d_{-}=\frac{\ln \left(S_{0} / K\right)+\left(r-(1 / 2) \check{\sigma}_{\rho} t\right)}{\check{\sigma}_{\rho} \sqrt{t}},
\end{aligned}
$$

$\Phi(x)$ is a normal distribution and $\check{\sigma}_{\rho}$ is defined in (3.21).

\subsection{European Call Option Pricing Formula for Merged Diffusion GMRP}

From Section 3.2, the merged diffusion GMRP has the following form:

$$
\frac{d \widetilde{S}(t)}{\widetilde{S}(t)}=\frac{1}{2}\left(\widehat{\sigma}_{\rho}^{2}(\widehat{x}(t))-\widehat{\rho}_{2}(\widehat{x}(t))\right) d t+\widehat{\sigma}_{\rho}(\widehat{x}(t)) d w(t),
$$

where $\widehat{\sigma}_{\rho}^{2}$ and $\hat{\rho}_{2}$ are defined in Section 3.2 (see (3.18). Taking into account the result on European call option pricing formula for regime-switching geometric Brownian motion (see [4, page 224, corollary]), we obtain the option pricing formula for the merged diffusion GMRP:

$$
C=\int C_{T}^{\mathrm{BS}}\left(\left(\frac{z}{T}\right)^{2^{-1}}, T, S_{0}\right) F_{T}^{x}(d z)
$$

where $C_{T}^{\mathrm{BS}}$ is a Black-Scholes value and $F_{T}^{x}(d z)$ is a distribution of the random variable

$$
z_{T}^{x}=\int_{0}^{T} \widehat{\sigma}_{\rho}^{2}(\widehat{x}(t)) d s
$$

where $\widehat{x}(t)$ is a merged Markov process. 


\section{Acknowledgment}

This research is partially supported by the University of Prince Edward Island major research grants (MRG) of M. S. Islam and NSERC grant of A. Swishchuk.

\section{References}

[1] A. Swishchuk, Random Evolutions and Their Applications: New Trends, vol. 504 of Mathematics and Its Applications, Kluwer Academic Publishers, Dordrecht, The Netherlands, 2000.

[2] A. V. Swishchuk and M. S. Islam, "The geometric Markov renewal processes with application to finance," Stochastic Analysis and Application, accepted.

[3] V. Korolyuk and A. Swishchuk, Evolution of Systems in Random Media, CRC Press, Boca Raton, Fla, USA, 1995.

[4] A. Swishchuk, Random Evolutions and Their Applications, vol. 408 of Mathematics and Its Applications, Kluwer Academic Publishers, Dordrecht, The Netherlands, 1997.

[5] K. K. Aase, "Contingent claims valuation when the security price is a combination of an Itô process and a random point process," Stochastic Processes and Their Applications, vol. 28, no. 2, pp. 185-220, 1988.

[6] J. C. Cox, S. A. Ross, and M. Rubinstein, "Option pricing: a simplified approach," Journal of Financial Economics, vol. 7, no. 3, pp. 229-263, 1979.

[7] A. Skorokhod, "Studies in the theory of random processes," The Annals of Mathematical Statistics, vol. 38, no. 1, 1967.

[8] F. Black and M. Scholes, "The pricing of options and corporate liabilities," Journal of Political Economy, vol. 81, pp. 635-654, 1973. 


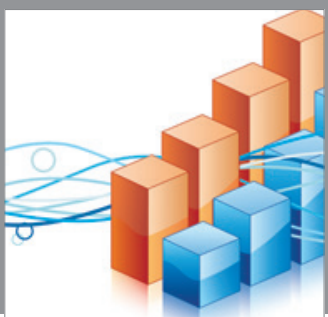

Advances in

Operations Research

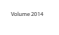

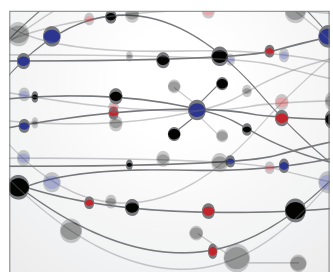

\section{The Scientific} World Journal
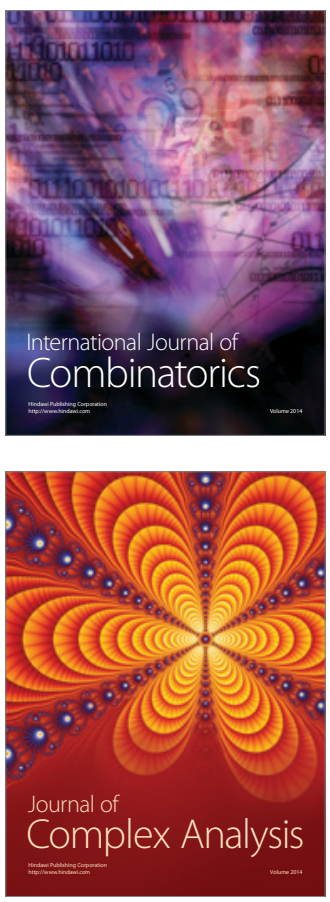

International Journal of

Mathematics and

Mathematical

Sciences
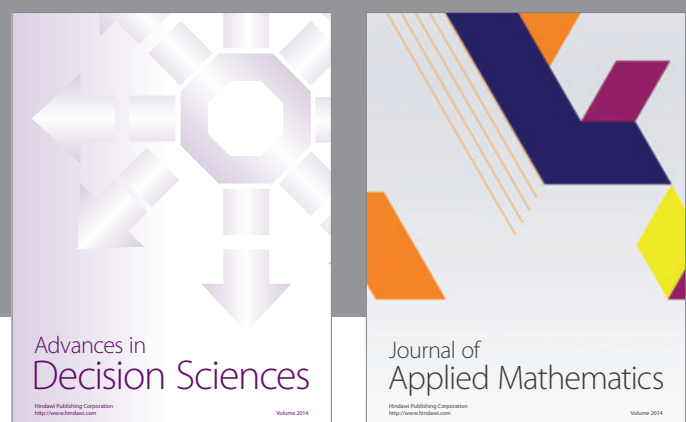

Journal of

Applied Mathematics
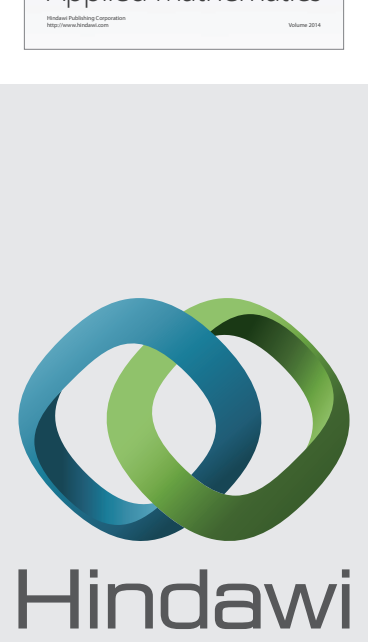

Submit your manuscripts at http://www.hindawi.com
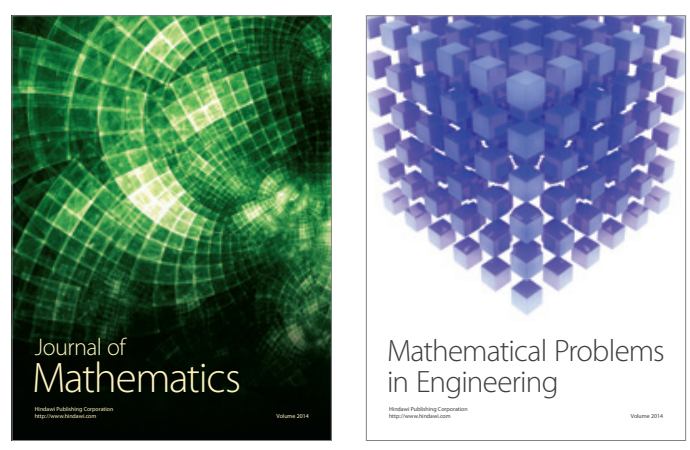

Mathematical Problems in Engineering
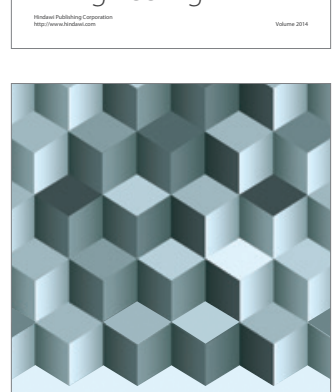

Journal of

Function Spaces
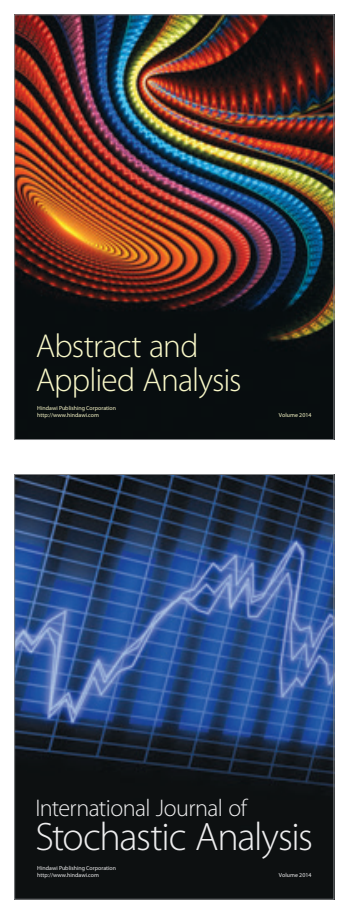

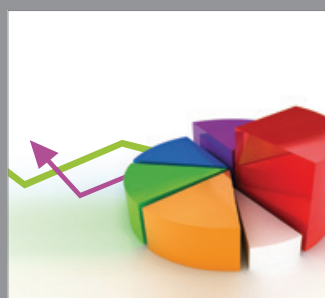

ournal of

Probability and Statistics

Promensencen
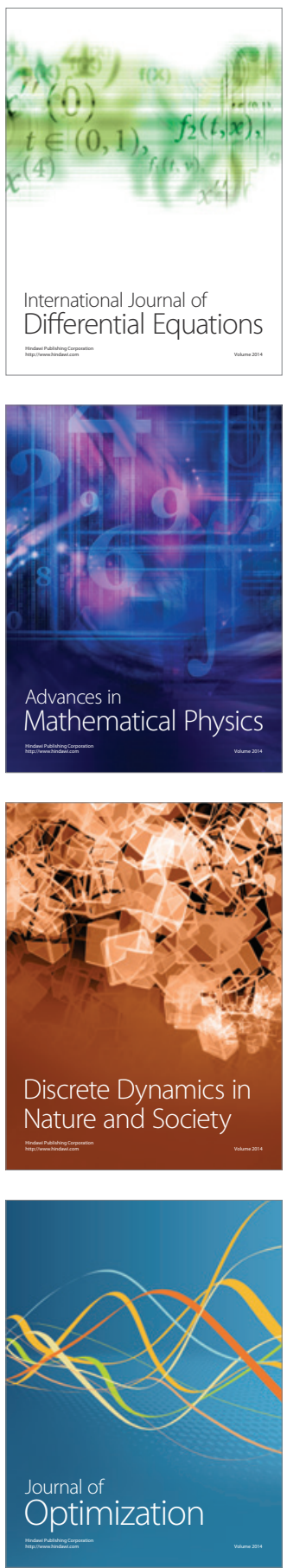